\title{
IDENTIFICATIONS OF FAINT IRAS SOURCES
}

\author{
M.W. KÜMMEL, S.J. WAGNER \\ Landessternwarte Heidelberg \\ Königsstuhl 12 \\ 69117 Heidelberg \\ Germany
}

From overlapping scans in the IRAS all-sky survey and additional pointed observations the deepest far infrared survey before ISO exists in the region around the North Ecliptic Pole (NEP) (Hacking P. and Houck J.R., ApJS 63 p. 311). This survey contains detections up to 10 and fluxes up to 100 times fainter than the IRAS survey. In the central square degree around the NEP we combine the far IR-survey with deep radio data at $151 \mathrm{MHz}$ and $1.5 \mathrm{GHz}$ (Visser, A.E. et al., A\&AS 110 p. 419, Kollgaard, R.I. et al., ApJS 93 p. 145) and own observation at $2.2 \mu m$ (K') and $435 \mathrm{~nm}(\mathrm{~B})$. The error circle around the IRAS source was chosen to include the true source with $85 \%$ probability (1.4 sigma). For 29 of the 32 IRAS sources we found at least one possible counterpart. Ten of the objects have multiple (up to four) counterparts in K'. Four of the IRAS sources have counterparts in the $1.5 \mathrm{GHz}$ survey. The higher accuracy of the radio position $\left(\sim 1^{\prime \prime}\right)$ allowed an unambiguous identification of the K' counterpart. None of the IRAS sources could be found in the $151 \mathrm{MHz}$ survey. The broad band spectra of the three galaxies with measured radio flux exhibit maximum emission between the radio band and $100 \mu m$ which corresponds to emission by cool dust ( $<50 \mathrm{~K})$. Contrary to the infrared luminosity functions at $12 \mu \mathrm{m}$ and $60 \mu \mathrm{m}$ which show power laws, the K' luminosity function is bimodal. The brightest K' objects are all point sources. Due to the small number statistics the power law indices of the luminosity functions can not be distinguished. We find a linear relationship between the K' flux and the flux at $60 \mu \mathrm{m}$ and $12 \mu \mathrm{m}$ over at least one decade. The large deviations by individual sources make an identification of the correct counterpart through this relation impossible. The spectral energy distributions of unambiguously identified sources span only one decade in energy $\left(\nu S_{\nu}\right)$, i.e. they have flat energy distributions. This suggests an identification of K' objects with flat energy distribution in case of multiple counterparts. 